\title{
Isoprinosine $^{\mathrm{n}}$ en la hepatitis viral aguda tipo $\mathrm{A}$
}

\section{Comunicación preliminar.}

Dr. Eduardo Welch W.l Dr. Santiago Rubio A.
Dr. Sergio Ceresa O' Jimena Guerrero R. ${ }^{3}{ }^{4}$ Dr. Jorge Las Heras 5 Dra. Maria Adela Godoy R.6

Treatment of type a viral hepatitis with isoprinosine ${ }^{\mathrm{P}}$

\begin{abstract}
Isoprinosine (R), (dimethylaminoisopropanol p-acetamielobenzoic acid sult: inosine 3: Imolar ratio), a drug with antiviral pmperties was tested in a double blind prokocol in thirteen children with type $\mathrm{A}$ viral (Placebo $\mathrm{n}$ : six; Drug n: seven) hepatítis along fifteen days from the beginning of dnug therpy, no significant differences were detected in hemograns, liver enzime tests, serum bilimubin, uric acid and hepatic histology between both groups of patients at days one, seven and fifteen of treutment.
\end{abstract}

El progresivo aumento de las notificaciones $y$ hospitalizaciones de casos de Hepatitis Viral Aguda Tipo A, fenómeno que se estaría presentando a nivel mundfal, plantea una serie de problemas de indole médico, soctal y epideniológico.

Conociendo las características especiales de la infección viral, esencialmente en loque se refiere a su condición de parásito intracelular estricto, se hace muy dificil un trataniento específico efectivo, que sea inocuo para el huésped. ${ }^{1.4}$

La Unidad de Enfemedades Infecciosas del Servicio de Pediatría, del Hospital Paula Jaraquemada, no es ajena a esta situación, motivo por el cual, revisando la literatura ${ }^{1-5}$ y tratasido de obtener una mejoría más pronta de la función hepática de nuestros pacientes y su reincorporación a las actividades escolares, nos decidimos a estudiar el efecto de la droga P-acetamidobenzoato de dimetilaminopropanol-inosina (Isoprinosine), en la Hepatitis Aguda Viral Tipo A.

El medicamento antes mencionado fue sintetizado a partir de un complejo químico inosinalquiloaminoalcohol, hace más o menos 17 años, en Estados Unidos, por el científico norteamericano Dr. Paul Gordon (Chicago Medical School), quien

\footnotetext{
${ }^{1}$ Jefe Unidad de Enfennedades Infecciusus, Hospibal Paula Jaraquemada.

${ }^{2}$ Jefe Clínica Servicio de Pediatria. Hospital Paula Jaraquemada.

${ }^{3}$ Académico Universidad de Chile, Facultad de Ciencias Médicas, División Sur, Departamento de Pediatria

4Servicio de Gastroenterología Infantil. Hospital Paula Jaraguemada.

Jutútomo Patólogo Intantil, Hospital Paula Jaraquemada.

${ }^{6}$ Becado Servicio de Pediatria Hospital Paula Jaraquemada.
}

demostró actividad antiviral in vitro e in vivo de esta sustancia; el uso en el ser humano se inició como estudio piloto en Argentina ${ }^{6}$ y Filipinas, generalizándose luego su uso en el resto del mundo, en diferentes afecciones virales.

En este momento se conocen las siguientes caracteristicas de la droga: $:^{4,7}$

Acción antiviral propia.

Potenciación de la respuesta humoral y celular.

Respeta la integridad histológica y fisiológica de la célula humana.

Al ser administrada por via oral se absorbe más o menos en 20 minutos, alcanzando la mayor concentración entre la segunda y la tercera hora.

Como efecto colateral importante se describe alza moderada del ácido úrico, que vuelve a valores normales al suspender el medicamento.

Necesita, para actuar eficazmente, que el sistema inmune esté indemne: se ha visto que en ratas tratadas con cortisol o inmunosupresores más Isoprinosine en forma simultánea, la acción terapéutica de éste último se anula.

\section{MATERLAL Y METODO}

El estudio se realizó en pacientes pediátricos hospitalizados con el diagnóstico de Hepatitis Viral Aguda Tipo A, no complicada, en la Unidad de Enfermedades Infecciosas del Servicio de Pediatría, del Hospital Paula Jaraquemada, entre los meses de octubre 1980 y septiembre de 1981.

El diagnóstico de Hepatitis Viral Aguda Tipo A, se hizo por descarte, tomando en cuenta la alta prevalencia de este virus en nuestro país y en la edad pediátrica, en ausencia de antecedentes de 
transfusiones y con resultados negativos en la investigación del antígeno de superficie $\mathrm{HB}_{5} \mathrm{Ag}_{\mathrm{g}}$, único métado para identificación de virus de Hepatitis con que se contaba en ese momento.

La muestra estaba formada por 13 niños ( 7 para la droga y 6 para el placebo), que cumplian con las condiciones de:

Evolución inferior a 4 semanas, no haber recibido terapia previa enfocada al hígado, no tener enfermedades virales concomitantes y contar con la autorización escrita de los padres, para puder efectuar la biopsia hepática, previa explicación del motivo y riesgos del procedimiento. No se consìderó edad, sexo, peso ni gravedad del paciente.

Se eligió el sistema de doble ciego para la administración del medicamento, que se entregó en forma alternada con el placebo, usando para la tabulación de los datos la denominación de Isoprinosine A e lsoprinosine B, abriéndose el doble ciego después de haber realizado el análisis estadístico.

Por contar con un número reducido de casos se utilizó para el análisis estadístico la distribución "T" de Student, con un nivel de significación de $5 \%$ y para que ambos grupos fuesen comparables se retiró del grupo Isoprinosine el caso cuyos valores se acercaban más al promedio.

El grupo catalogado como Isoprinosine A, recibió la droga y estaba formado por 7 niños entre 10 años y 4 años, con una edad promedio de 5.4 años, en relativo buen estado nutritivo, sin enfermedades concomitantes y que provenían del Area Certral de Santiago.

El grupo catalogado como Isoprinosine B, recibió placebo y se componia de 6 niños de igual edad media, estado nutritivo y procedencia, uno de los cuales se retiró del protocolo después de encontrarse, en la primera biopsia hepática, elementos de Hepatitis Colestásica, pero como el estudio de laboratorio se alcanzó a terminar antes de tener el resultado de la biopsia se mantuvo para efectos de análisis estadístico.

Para tabular los casos se diseñó un protocolo que andiza caracteristicas generales del paciente: edad, fecha, procedencia, exámenes generales realizados en forma seriada los días $1 . .^{\circ}, 7 .^{\circ}$ y $14 .^{\circ}$ de hospitalización $y$, resultado de las biopsias hepáticas realizadas al $I .^{\circ}$ y $150^{\circ}$ días de hospitalización. (Fig. 1).

El examen de las biopsias hepáticas se hizo basándose en 6 parámetros: desorganización de la

Figura 1

Hepatitis Aguda Viral. Estudio Isoprinosine

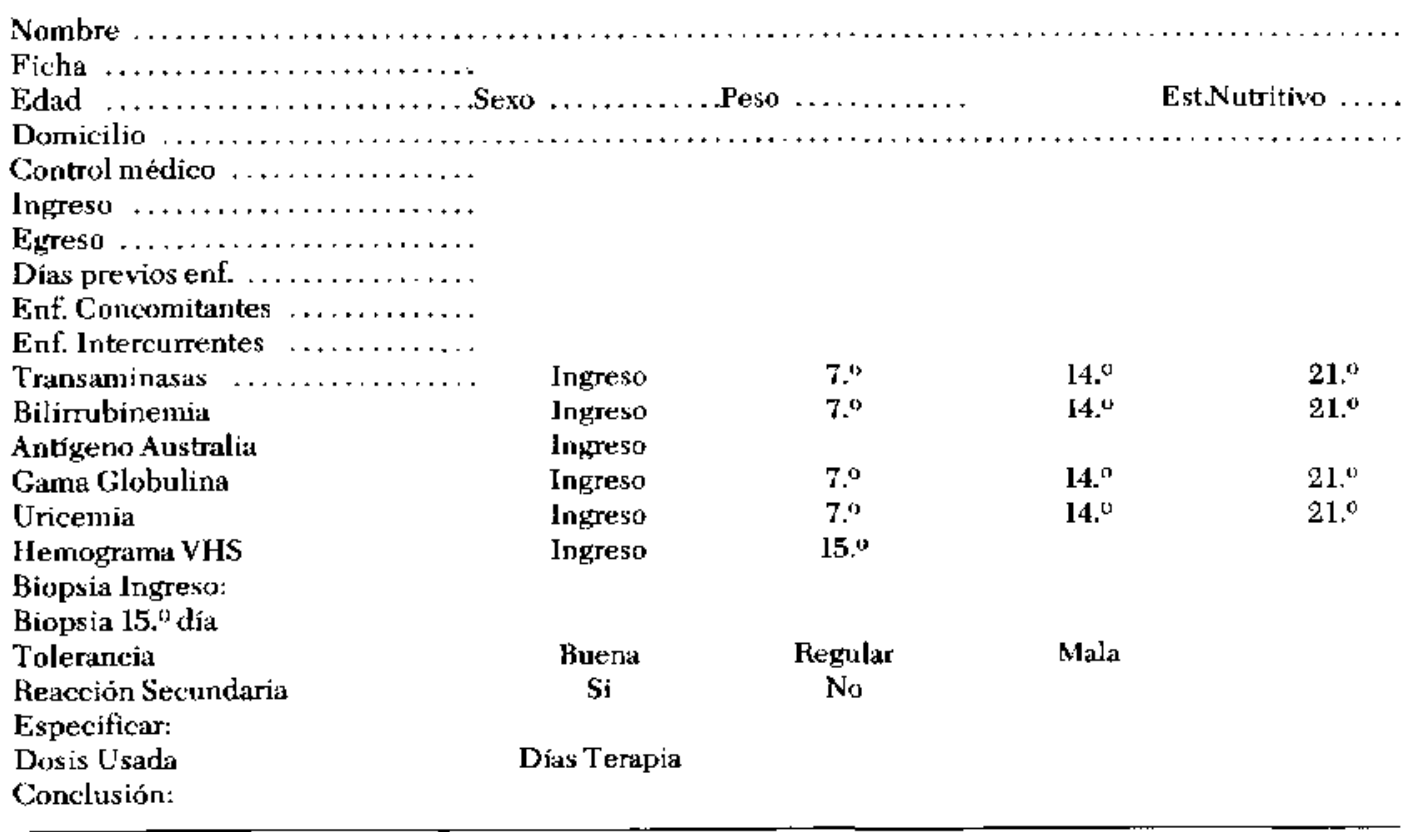


Tabla 1

Fxámenes de función hepática en pacientes tratados con isoprinosine y con placebo.

\begin{tabular}{|c|c|c|c|c|c|c|c|c|c|c|c|c|c|c|c|c|c|c|}
\hline \multirow{3}{*}{ Casos } & \multicolumn{6}{|c|}{ Transaminasas } & \multicolumn{6}{|c|}{ Bilimubina } & \multicolumn{6}{|c|}{ Fosfatasas Alcalinas } \\
\hline & \multicolumn{2}{|c|}{ I } & \multicolumn{2}{|c|}{ VII } & \multicolumn{2}{|c|}{ XIV } & \multicolumn{2}{|r|}{ I } & \multicolumn{2}{|c|}{ VII } & \multicolumn{2}{|c|}{ XIV } & \multicolumn{2}{|c|}{ I } & \multicolumn{2}{|c|}{ VII } & \multicolumn{2}{|c|}{ XIV } \\
\hline & Droga & Placebo & Droga & Placebo & Droga & Placebo & Droga & Placebo & Droga & Placebo & Droga & Placebo & Droga & Placebo & Droga & Placebo & Droga & Placebo \\
\hline 1 & 2000 & 1300 & 73 & 90 & 63 & 55 & 5.1 & 4.6 & 1.26 & 0.42 & 0.9 & 1.1 & 21.6 & 11.8 & 19.6 & 6.2 & 10.4 & 7 \\
\hline 2 & 232 & 1900 & 142 & 230 & 67 & 52 & 3.4 & 5.6 & 0.42 & 0.42 & 0.84 & 2.1 & 17.2 & 14.9 & 10.7 & 12.1 & 7.9 & 9.1 \\
\hline 3 & 690 & & 63 & 72 & 45 & 28 & 9.4 & 6.3 & 1.2 & 1.2 & 1.68 & 1.2 & 7.4 & 18.2 & 4.6 & 8.1 & 4.2 & 6.4 \\
\hline 4 & 142 & 258 & 67 & 103 & 61 & 132 & 4.3 & 8.4 & 0.0 & 6.7 & & 3.36 & 39.2 & 19 & 26.9 & 28 & 24 & 23.7 \\
\hline 5 & 2000 & 195 & 59 & 160 & 60 & 51 & 4.4 & 7.14 & 1.93 & 2.52 & 0.92 & 1.2 & 13.4 & 8.7 & 9 & 7.8 & 5.6 & 7.1 \\
\hline 6 & 1090 & 300 & 68 & 150 & 36 & 43 & 2.18 & 7.14 & 0.97 & 1.76 & 0.92 & 1.68 & 26.3 & 28 & 12.6 & 28 & 11.3 & 11.8 \\
\hline $\bar{X}$ & 1025 & 925.5 & 78.67 & 134.17 & 55.33 & 60.1 & 4.80 & 6.53 & 0.96 & 2.17 & 1.06 & 1.77 & 20.85 & 16.76 & 13.9 & 15.03 & 8.9 & 10.85 \\
\hline
\end{tabular}

\begin{tabular}{|c|c|c|c|c|c|c|c|c|}
\hline $\begin{array}{l}\overline{\mathrm{D}}=100,17 \\
D_{5}=1259 \\
D>0.05\end{array}$ & $\begin{array}{l}\bar{D}=-55,50 \\
D_{5}=39.63 \\
p>0.05\end{array}$ & $\begin{array}{l}\overrightarrow{\mathrm{D}}=864.8 \\
\mathrm{D}_{s}=781.1 \\
\mathrm{p}>0.05\end{array}$ & $\begin{array}{l}\overline{\mathrm{D}}=\cdot 1.73 \\
\mathrm{D}_{\mathrm{s}}=3.01 \\
\mathrm{p}>0.05\end{array}$ & $\begin{array}{l}\bar{D}=1.207 \\
D_{s}=7.57 \\
p>0.05\end{array}$ & $\begin{array}{l}\vec{D}-0.71 \\
D_{5}=1.78 \\
p>0.05\end{array}$ & $\begin{array}{l}\bar{D}=4.09 \\
D_{s}=10.5 \\
p>0.05\end{array}$ & $\begin{array}{l}\bar{D}=-1.13 \\
D=9.23 \\
p>0.05\end{array}$ & $\begin{array}{l}\bar{D}=-1.95 \\
D-4.2 \\
p>0.05\end{array}$ \\
\hline
\end{tabular}


arquitectura, inflamación periportal, fibrosis portal, colestasia intracanicular, necrosis hepatocitaria y colestasia hepatocitaria.

El medicamento se administró en dosis de 75 $\mathrm{mg} / \mathrm{Kg} / \mathrm{dia}$, fraccionado en 5 dosis (c/4 hrs.) diarias por vía oral, durante 10 días.

Los exámenes de función hepática (transaminasas, fosfatasas alcalinas, gamaglutamiltransferasa, bilirrubina y protrombina), hemograma uricemia, HBsAg, se realizaron en el Laboratorio de nuestro Hospital, usando técnicas standard; el examen de las biopsias fue hecho por un solo médico para evitar errores de tipo subjetivo.

Finalmente se realizó una correlación entre los datos recogidos y el uso de la droga y el placebo.

\section{RESULTADOS}

Un paciente del grupo Placebo debió retirarse del trabajo por encontrarse, en la primera biopsia, características de Hepatitis Colestásica, requiriéndose el uso de terapia esteroidal.

El descenso de las transaminasas, bilimubina, fosfatasas alcalinas, fue similar en los dos gnupos,

\section{Tabla 2}

Resultados de biopsia hepática en hepatitis aguda viral tratada con isoprinosine y placebo

\begin{tabular}{|c|c|c|c|c|}
\hline \multirow[t]{2}{*}{ Alteración } & \multicolumn{2}{|c|}{$\begin{array}{c}\text { Biopsia } \\
\text { Isoprinosine }\end{array}$} & \multicolumn{2}{|c|}{$\begin{array}{l}\text { Biopsia } \\
\text { Placebo }\end{array}$} \\
\hline & I & II & $\mathbf{I}$ & II \\
\hline $\begin{array}{l}\text { Desorganización de } \\
\text { la arguitectura }\end{array}$ & $* 7 \pi$ & $4 / 7$ & $5 / 6$ & 255 \\
\hline $\begin{array}{l}\text { Inflamación } \\
\text { Periportal }\end{array}$ & $7 / 7$ & $4 / 7$ & $5 / 6$ & $3 / 5$ \\
\hline Fibrosis portal & $3 / 7$ & $2 / 7$ & $1 / 6$ & $1 / 5$ \\
\hline $\begin{array}{l}\text { Colestasia } \\
\text { Intracanalicular }\end{array}$ & or & $0 / 7$ & $1 / 6$ & \\
\hline $\begin{array}{l}\text { Necrosis } \\
\text { Hepatocitaria }\end{array}$ & $6 / 7$ & $4 / 7$ & $5 / 6$ & $2 / 5$ \\
\hline $\begin{array}{l}\text { Colestasia } \\
\text { Hepatocitaria }\end{array}$ & $5 / 7$ & $3 / 7$ & $4 / 6$ & $2 / 5$ \\
\hline
\end{tabular}

*N." de casos / N. " total de casos.

$\mathbf{P}=$ NS. no encontrándose diferencias estadísticamente significativas $\mathrm{p}<0.05$. (Tabla 1).

Al examinar las biopsias se encontro (Tabla 2) desorganización de la arquitectura e inflamación periportal en todos los pacientes del estudio, fibrosis portal en un paciente del grupo Placebo y tres del grupo Droga, Colestasia intracanalicular en un solo paciente del grupo Placebo que se retiró del estudio, necrosis hepatocitaria en todos los pacientes del grupo Placebo y tres del grupo Droga y colestasia hepatocitaria en cuatro pacientes del grupo Droga y tres del grupo Placebo. Estas alteraciones prácticamente no se modificaron en la segunda biopsia.

No se detectaron reacciones secundarias al uso del medicamento y las determinaciones seriadas de Uricemia dieron siempre valores normales. (Tabla 3).

No se encontraron variaciones en la serie de hemogramas, gamaglutamiltransferasas (que sólo pudo realizarse en algunos casos en forma seriada). El tiempo de protrombina no se tabuló por existir factores de dispersión individual demasiado amplios. (Tabla 3).

\section{COMENTARIO}

A pesar de que en la literatura extranjera se describen resultados promisorios en la Hepatitis Viral Aguda Tipo A tratada con Isoprinosine, principalmente nomalización de las transaminasas y mejoría del estado general, pero sin biopsia hepática comprobatoria, nos parece discutible su uso en el momento actual.

Cabe hacer notar que actualmente se pueden estudiar, mediante método de Radioinmunoensayo, marcadores de los virus de la hepatitis que permiten su clasificación en Hepatitis A, no A, no B y B; exámenes con los cuales no se contaba al iniciar este estudio.

Nuestra experiencia no reveló diferencias significativas entre el placebo y el Isoprinosine, por método de doble ciego, en ninguno de los pacientes estudiados durante el tratamiento de la Hepatitis Viral Aguda Tipo A.

Creemos que, pese al pequeño tamaño de la muestra y al estudio virulógico limitado, es posible concluir que no se obtuvo evidencia de un efecto útil de la droga sobre la evolución de la enfermedad. 
Comparación de promedius de valores seriados de hemograma uricemia y gamaglutamiltransferasa

\begin{tabular}{|c|c|c|c|c|c|c|c|c|c|c|}
\hline \multirow{3}{*}{ Casos } & \multicolumn{6}{|c|}{ Hemograna } & \multicolumn{2}{|c|}{ Uricemia } & \multicolumn{2}{|c|}{ Gamaglutamiltransferasa } \\
\hline & \multicolumn{3}{|c|}{ lsoprinosine } & \multicolumn{3}{|c|}{ Placebo } & \multirow[t]{2}{*}{ Isoprinosine } & \multirow[t]{2}{*}{ Placebo } & \multirow{2}{*}{$\begin{array}{c}\text { lsoprinosine } \\
109.5\end{array}$} & \multirow{2}{*}{$\begin{array}{c}\text { Placebo } \\
99.6\end{array}$} \\
\hline & Hto. & $\begin{array}{l}\text { Linfo- } \\
\text { citos }\end{array}$ & $\begin{array}{l}\text { Pla- } \\
\text { que- } \\
\text { tas }\end{array}$ & He. & $\begin{array}{l}\text { Linfo- } \\
\text { citos }\end{array}$ & $\begin{array}{l}\text { Pla- } \\
\text { que- } \\
\text { tas }\end{array}$ & & & & \\
\hline 1 & 38 & 53.5 & $N$ & 42 & 5.5 .5 & $N$ & 3.3 & 3.96 & Incompleto & 249.6 \\
\hline 2 & 38 & 50.5 & $\mathbf{N}$ & 35 & 57 & $N$ & 2.4 & 2.87 & 18.9 & Incompleto \\
\hline 3 & 40 & 40 & $\mathbf{N}$ & 36.5 & 51 & $N^{-}$ & 2.6 & 1.98 & 135 & 182.3 \\
\hline 4 & 33.5 & 37.5 & N & $3 \vec{i}$ & 64 & $N$ & 4.6 & 3 & 244 & 232.6 \\
\hline 5 & 38.5 & 34.5 & $\mathrm{~N}$ & 3) & 53 & $N$ & 2.3 & 2.26 & Incompleto & 169.3 \\
\hline 6 & 35 & 58 & $\mathrm{~N}$ & 36.5 & 40 & $N$ & 2.0 & 3.6 & Incompleto & 186 \\
\hline $\mathbf{x}$ & 37.1 & 45 & & 36.1 & 53.4 & & 2.86 & 2.94 & & \\
\hline
\end{tabular}

RESUMEN

Ante el aumento progresivo de la Hepatitis Viral Aguda Tipo A y la necesidad de aminorar la magnitud del impacto médico social, económico, laboral, de ausentismo escolar y factores epidemiológicos de esta enfermedad. Se estudió el efecto del Isoprinosine sobre la evolución clínica, histológica y de laboratorio en 13 niños sin demostrar ventajas con respecto al uso de placebo.

\section{AGRADECIMIENTOS}

Al Laboratorio Farmoquimica del Pacifico el haber proporcionado la droga y el placebo.

\section{REFERENCIAS}

${ }^{1}$ Newport Pharmaceutical International, Inc: Inmunoterapia. El Umbral Dramático. Agosto 197.

${ }^{2}$ Glasky A.J. a al.: Isoprinosine (R) a Purine Derivative Metabolic, Inumological, and Antiviral Effects Combines Inmunodeficiency, Birth Defects Institute. Symposium IV October 3-3. 1973.

${ }^{3}$ Ginsberg T.: Excreción uritaria de metabolitiss de purina en mucaca mulatta después de la administración de inosine y I(dimetilamino 1-2- propunol, p-acetatomidobenzodto. Vth. International Congress on Pharmacology. 1977-82. Extracto.

4 Glasky A.J. ef al.: Limphocyte Response to Mitogens. Int'L Congress on Immumology Meeting in Australia, July 1971. Abstract.

${ }^{5}$ Friebertshaver, G.E, et ad: Kelationships between Molecular Biochemical Effects of Isoprinosine and Itmonunological Pherionena. Publication of the 1974 Pacific Slope Biochemical Conterence. Fiverside California August 26-28, 1974.

${ }^{6}$ Ink, J. et a!.: Estudio clínico bioestadístico de diterentes virosis tratadis con p-acetamidobenzoato de dimetilaminopropanol inosina. Prensa médica Argentiná 58: 1875- 1890, 1971.

${ }^{7}$ Ginsbeng, T. Glashy, A.J., Inostplex: An Immumomodulution Model for the treabiuent of viral Disease. $3 \mathrm{rd}$ Conterence on Antiviral Substances, X.Y. Acaderny of Sciences. Feb. 1976. 